\title{
Effect of Different Spore Concentration of Entomopathogenic Fungi Isolates against Mealy Bug Maconellicocus hirsutus
}

\author{
S.B. Sable ${ }^{1 *}$, P.B. Deore ${ }^{1}$, V.R. Bangar ${ }^{2}$ and K.C. Kurhade ${ }^{2}$ \\ ${ }^{1}$ College of Agriculture, Dhule, Plant Pathology Section, MPKV, Rahuri, Maharashtra, India \\ ${ }^{2}$ Department of Plant Pathology, PGI, MPKV, Rahuri, Maharashtra, India \\ *Corresponding author
}

\begin{abstract}
A B S T R A C T
Keywords

Entomopathogenic fungi, Cadaver,

Aspergillus

Spp., Isolates,

Virulent,

Concentration,

Maconellicoccus

hirsutus

Article Info

Accepted:

18 February 2019

Available Online:

10 March 2019

Entomopathogenic fungi suppress the diverse group of insect pest such as coleopterans, lepidopterous and sucking pest. Wide spread incidence of Aspergillus niger, Aspergillus flavus and Aspergillus tamarii was observed during extensive and repeated survey covering different agro ecological niches of Dhule, Nandurbar, Jalgaon, Nasik and Beed districts of Maharashtra (India) on insect cadavers infected with fungi. From the insect cadavers the fungal isolates were isolated on PDA media. Out of nineteen samples collected only fifteen samples showed the growth of fungus on DOC 2-50\% selective media. Virulence of entomopathogenic fungi isolates against mealy bugs (Maconellicoccus hirsutus) revealed that as the spore concentration increased from $10^{-3}$ to $10^{-9}$ the insect mortality was decreased. Among all the fifteen isolates the highest insect mortality was recorded at $10^{-3}$ spores $/ \mathrm{ml}$ concentration and the spore dilution of entomopathogenic fungi isolates increased from $10^{-3}$ to $10^{-9}$ spores $/ \mathrm{ml}$ the time required for insect mortality was also increased. Among the isolates of entomopathogenic fungi the isolate EPF-14 was the most virulent. Among each isolate, it was observed that as the spore dilution increased the insect mortality decreased. Aspergillus tamarii, A. niger and A. flavus the isolate EPF-7, EPF-14 and EPF-6 were the most virulent, respectively against mealy bugs at $10^{-3}$ spores/ml concentration and isolates EPF-1, EPF-2 and EPF-6 were the most virulent isolates respectively against mealy bugs at $10^{-6}$ and $10^{-9}$ spores $/ \mathrm{ml}$ concentration.
\end{abstract}

\section{Introduction}

Many genera of entomopathogenic fungi are being used in agricultural crop for pest management such as Lower fungi i.e. Mastigomycotina, Ascomycotina, Basidomycotina and fungi imperfecti which includes several genera like Aspergillus, Beauveria, Metarhizium, Nomuraea, Paecilomyces, Penicillium, Trichoderma, Verticillium etc. which suppress the diverse group of insect pest such as coleopterans, lepidopterous, sucking pest. Amongst these, several asexual stages of fungi are associated with insect infection. Pasteur (1874) was one of the first to suggest that microorganisms could be used to control insect pests. Numerous groups of entomopathogenic fungi were described during the $19^{\text {th }}$ century. One of the earliest successes in biocontrol was the use of Aschersonia aleyrodes to control citrus white flies in Florida (Berger, 1921). 
Microbial control of crop pest offers an environmentally acceptable strategy with lower cost and effect in longer run. Entomopathogenic fungi played a significant role in the history of insect pathology and especially in microbial control (Steinhous, 1956). There are approximately 750 species of fungi from 56 genera that infect arthropods. These are ubiquitous and in appropriate hosts are capable of natural recycling (Hajek and Leger, 1994; Alexopoulos et al., 1996).

Recently increased use of conventional chemical pesticides over the years has not only contributed to an increase in food production, but also has resulted in adverse effects on the environment and non-target organisms. In view of these side effects, the necessity for sustainable crop production through eco-friendly pest management technique is being largely felt in the recent times. Hence, the present investigation was planned and carried out encompassing to study the virulence of different isolates of entomopathogenic fungi against mealy bugs Maconellicoccus hirsutus.

\section{Materials and Methods}

\section{Survey}

The field survey was conducted in Dhule, Nandurbar, Jalgaon, Nasik and Beed districts of Maharashtra (India) during kharif, 2014 to collect the insect cadavers from fields and forest areas. During the survey, different locations were surveyed and nineteen insect cadavers infected with fungus were collected and placed in separate plastic containers of 6 $\mathrm{x} 4 \mathrm{~cm}$ size.

\section{Isolation of entomopathogenic fungi}

The selective media DOC2-50\% (Shin et al., 2010) was prepared for the isolation of pure cultures entomopathogenic fungi. The infected portion of each insect cadaver was cut into small bits and a small portion of infected tissue was transferred aseptically to a culture plate containing DOC2-50\% selective media. The inoculated culture plates were incubated at $28 \pm 2{ }^{\circ} \mathrm{C}$ in BOD incubator and kept under constant observation for the growth and development of fungus. Three to five days after incubation, the fungus growth was purified by sub-culturing and slants of each purified fungus culture were prepared.

\section{Identification of entomopathogenic fungi isolates}

The purified coded fungus isolates were sent to Indian Type Culture Collection, Division of Plant Pathology, Indian Agricultural Research Institute, New Delhi - 110012 for identification.

\section{Preparation of spore inoculum and inoculation of insects}

For the preparation of spore inoculum, the fifteen days mature old culture grown on PDA media were used. The spores of each fungal isolate were harvested from PDA plates and the spore suspensions of $10^{-3}, 10^{-6}$ and $10^{-9}$ spores $/ \mathrm{ml}$ concentration of each fungus isolate were prepared by mixing harvested spores with distilled water and 0.2 per cent Tween80. The spore concentration was determined by using a Neubauer hemocytometer. The spore suspensions of all isolates were applied by direct dipping method on twenty-five adult mealy bugs per treatment (isolate) and experiment was replicated twice.

The adult mealy bugs were dipped in spore suspension for 30 seconds. The inoculated mealy bugs were placed on surface sterilized sprouted potato and red pumpkin in laboratory at room temperature. Thereafter, inoculated mealy bugs were checked daily and mortality was recorded. 


\section{Virulence of entomopathogenic fungi}

For the virulence study the entomopathogenic fungi isolates were evaluated at different spore concentration against mealy bugs and insect mortality was observed at $24 \mathrm{hr}$ interval after inoculation up to 10 days. The percent mortality was calculated by using following formula.

Total no. of dead

$\begin{array}{lcc}\text { Percent } & \text { mealy bug } & \text { X } \\ \text { mortality }= & \text { Total no. of } & 100 \\ \text { inoculated mealy } & \text { bug }\end{array}$

\section{Results and Discussion}

During the survey, different locations were surveyed and nineteen insect cadavers infected with fungus were collected and brought to section laboratory. After fairly drying the collected insect cadaver samples were stored in refrigerator and coded for further study. Out of nineteen samples inoculated only fifteen samples showed the growth of fungus on DOC2-50\% selective media. As the identification report of Indian Type Culture Collection, Division of Plant Pathology, Indian Agricultural Research Institute, New Delhi the coded entomopathogenic fungal isolates were decoded as follows (Table 1).

The virulence studies of entomopathogenic fungi isolates against mealy bugs (Maconellicoccus hirsutus) revealed that as the spore concentration increases from $10^{-3}$ to $10^{-6} \& 10^{-9}$ the insect mortality decreases. Among all the fifteen isolates the highest insect mortality was observed at $10^{-3}$ spores $/ \mathrm{ml}$ concentration. Also, it was observed that as the spore dilution of entomopathogenic fungi isolates increases from $10^{-3}$ to $10^{-9}$ spores/ $\mathrm{ml}$ the time required for insect mortality increases (Fig. 1).

Table.1 Identification of entomopathogenic fungi isolates

\begin{tabular}{|c|l|l|c|}
\hline Sr. No. & $\begin{array}{c}\text { Isolate } \\
\text { Code }\end{array}$ & \multicolumn{1}{|c|}{ Fungus identified } & Remark \\
\hline $\mathbf{1}$ & EPF-1 & Aspergillus tamarii & EPF $^{*}$ \\
\hline $\mathbf{2}$ & EPF-2 & Aspergillus niger & EPF \\
\hline $\mathbf{3}$ & EPF-3 & Aspergillus niger & EPF \\
\hline $\mathbf{4}$ & EPF-4 & Aspergillus niger & EPF \\
\hline $\mathbf{5}$ & EPF-5 & Aspergillus tamarii & EPF \\
\hline $\mathbf{6}$ & EPF-6 & Aspergillus flavus & EPF \\
\hline $\mathbf{7}$ & EPF-7 & Aspergillus tamarii & EPF \\
\hline $\mathbf{8}$ & EPF-8 & Aspergillus flavus & EPF \\
\hline $\mathbf{9}$ & EPF-9 & Aspergillus flavus & EPF \\
\hline $\mathbf{1 0}$ & EPF-10 & Aspergillus niger & EPF \\
\hline $\mathbf{1 1}$ & EPF-11 & Aspergillus flavus & EPF \\
\hline $\mathbf{1 2}$ & EPF-12 & Aspergillus tamarii & EPF \\
\hline $\mathbf{1 3}$ & EPF-13 & Aspergillus flavus & EPF \\
\hline $\mathbf{1 4}$ & EPF-14 & Aspergillus niger & EPF \\
\hline $\mathbf{1 5}$ & EPF-15 & Aspergillus tamarii & EPF \\
\hline
\end{tabular}

*EPF=Entomopathogenic fungi 
Table.2 The virulence of entomopathogenic fungi isolates at $10^{-3}$ spore concentration

\begin{tabular}{|c|c|c|c|c|c|c|c|c|c|c|}
\hline \multirow[t]{2}{*}{ Isolate } & \multicolumn{10}{|c|}{ Per cent mortality at $10^{3}$ spore concentration } \\
\hline & 1DAT & 2DAT & 3DAT & 4DAT & 5DAT & 6DAT & 7DAT & 8DAT & 9DAT & 10DAT \\
\hline EPF-1 & $\begin{array}{c}0.00 \\
(0.00)\end{array}$ & $\begin{array}{c}0.00 \\
(0.00)\end{array}$ & $\begin{array}{c}0.00 \\
(0.00)\end{array}$ & $\begin{array}{c}32.00 \\
(34.45)\end{array}$ & $\begin{array}{c}32.00 \\
(34.45)\end{array}$ & $\begin{array}{c}42.00 \\
(40.40)\end{array}$ & $\begin{array}{c}42.00 \\
(40.40)\end{array}$ & $\begin{array}{c}62.00 \\
(51.94)\end{array}$ & $\begin{array}{c}62.00 \\
(51.94)\end{array}$ & $\begin{array}{c}70.00 \\
(56.80)\end{array}$ \\
\hline EPF-2 & $\begin{array}{c}0.00 \\
(0.00)\end{array}$ & $\begin{array}{c}0.00 \\
(0.00)\end{array}$ & $\begin{array}{c}0.00 \\
(0.00)\end{array}$ & $\begin{array}{c}8.00 \\
(16.43)\end{array}$ & $\begin{array}{c}24.00 \\
(29.33)\end{array}$ & $\begin{array}{c}34.00 \\
(35.67)\end{array}$ & $\begin{array}{c}38.00 \\
(38.06)\end{array}$ & $\begin{array}{c}44.00 \\
(41.55)\end{array}$ & $\begin{array}{c}70.00 \\
(56.79)\end{array}$ & $\begin{array}{c}70.00 \\
(56.79)\end{array}$ \\
\hline EPF-3 & $\begin{array}{c}0.00 \\
(0.00)\end{array}$ & $\begin{array}{c}0.00 \\
(0.00)\end{array}$ & $\begin{array}{c}16.00 \\
(23.58)\end{array}$ & $\begin{array}{c}16.00 \\
(23.58)\end{array}$ & $\begin{array}{c}16.00 \\
(23.58)\end{array}$ & $\begin{array}{c}40.00 \\
(39.23)\end{array}$ & $\begin{array}{c}64.00 \\
(53.13)\end{array}$ & $\begin{array}{c}64.00 \\
(53.13)\end{array}$ & $\begin{array}{c}76.00 \\
(60.67)\end{array}$ & $\begin{array}{c}80.00 \\
(63.44)\end{array}$ \\
\hline EPF-4 & $\begin{array}{c}0.00 \\
(0.00)\end{array}$ & $\begin{array}{c}0.00 \\
(0.00)\end{array}$ & $\begin{array}{c}0.00 \\
(0.00)\end{array}$ & $\begin{array}{c}20.00 \\
(26.56)\end{array}$ & $\begin{array}{c}20.00 \\
(26.56)\end{array}$ & $\begin{array}{c}20.00 \\
(26.56)\end{array}$ & $\begin{array}{c}34.00 \\
(35.67)\end{array}$ & $\begin{array}{c}40.00 \\
(39.23)\end{array}$ & $\begin{array}{c}40.00 \\
(39.23)\end{array}$ & $\begin{array}{c}72.00 \\
(58.05)\end{array}$ \\
\hline EPF-5 & $\begin{array}{c}0.00 \\
(0.00)\end{array}$ & $\begin{array}{c}0.00 \\
(0.00)\end{array}$ & $\begin{array}{c}0.00 \\
(0.00)\end{array}$ & $\begin{array}{c}24.00 \\
(29.33)\end{array}$ & $\begin{array}{c}24.00 \\
(29.33)\end{array}$ & $\begin{array}{c}50.00 \\
(45.00)\end{array}$ & $\begin{array}{c}50.00 \\
(45.00)\end{array}$ & $\begin{array}{c}50.00 \\
(45.00)\end{array}$ & $\begin{array}{c}82.00 \\
(64.90)\end{array}$ & $\begin{array}{c}82.00 \\
(64.90)\end{array}$ \\
\hline EPF-6 & $\begin{array}{c}0.00 \\
(0.00)\end{array}$ & $\begin{array}{c}0.00 \\
(0.00)\end{array}$ & $\begin{array}{c}0.00 \\
(0.00)\end{array}$ & $\begin{array}{c}12.00 \\
(20.27)\end{array}$ & $\begin{array}{c}12.00 \\
(20.27)\end{array}$ & $\begin{array}{c}30.00 \\
(33.21)\end{array}$ & $\begin{array}{c}30.00 \\
(33.21)\end{array}$ & $\begin{array}{c}50.00 \\
(45.00)\end{array}$ & $\begin{array}{c}50.00 \\
(45.00)\end{array}$ & $\begin{array}{c}58.00 \\
(49.60)\end{array}$ \\
\hline EPF-7 & $\begin{array}{c}0.00 \\
(0.00)\end{array}$ & $\begin{array}{c}0.00 \\
(0.00)\end{array}$ & $\begin{array}{c}16.00 \\
(23.58)\end{array}$ & $\begin{array}{c}16.00 \\
(23.58)\end{array}$ & $\begin{array}{c}36.00 \\
(36.87)\end{array}$ & $\begin{array}{c}36.00 \\
(36.87)\end{array}$ & $\begin{array}{c}36.00 \\
(36.87)\end{array}$ & $\begin{array}{c}64.00 \\
(53.13)\end{array}$ & $\begin{array}{c}64.00 \\
(53.13)\end{array}$ & $\begin{array}{c}74.00 \\
(59.34)\end{array}$ \\
\hline EPF-8 & $\begin{array}{c}0.00 \\
(0.00)\end{array}$ & $\begin{array}{c}0.00 \\
(0.00)\end{array}$ & $\begin{array}{c}0.00 \\
(0.00)\end{array}$ & $\begin{array}{c}0.00 \\
(0.00)\end{array}$ & $\begin{array}{c}20.00 \\
(26.56)\end{array}$ & $\begin{array}{c}26.00 \\
(30.66)\end{array}$ & $\begin{array}{c}54.00 \\
(47.29)\end{array}$ & $\begin{array}{c}54.00 \\
(47.29)\end{array}$ & $\begin{array}{c}54.00 \\
(47.29)\end{array}$ & $\begin{array}{c}80.00 \\
(63.44)\end{array}$ \\
\hline EPF-9 & $\begin{array}{c}0.00 \\
(0.00) \\
\end{array}$ & $\begin{array}{c}0.00 \\
(0.00) \\
\end{array}$ & $\begin{array}{c}0.00 \\
(0.00)\end{array}$ & $\begin{array}{c}0.00 \\
(0.00) \\
\end{array}$ & $\begin{array}{c}24.00 \\
(29.33) \\
\end{array}$ & $\begin{array}{c}26.00 \\
(30.66) \\
\end{array}$ & $\begin{array}{c}56.00 \\
(48.45) \\
\end{array}$ & $\begin{array}{c}56.00 \\
(48.45)\end{array}$ & $\begin{array}{c}70.00 \\
(56.79) \\
\end{array}$ & $\begin{array}{c}88.00 \\
(69.73) \\
\end{array}$ \\
\hline EPF-10 & $\begin{array}{c}0.00 \\
(0.00)\end{array}$ & $\begin{array}{c}0.00 \\
(0.00)\end{array}$ & $\begin{array}{c}0.00 \\
(0.00)\end{array}$ & $\begin{array}{c}0.00 \\
(0.00)\end{array}$ & $\begin{array}{c}24.00 \\
(29.33)\end{array}$ & $\begin{array}{c}24.00 \\
(29.33)\end{array}$ & $\begin{array}{c}54.00 \\
(47.29)\end{array}$ & $\begin{array}{c}54.00 \\
(47.29)\end{array}$ & $\begin{array}{c}86.00 \\
(68.03)\end{array}$ & $\begin{array}{c}90.00 \\
(71.56)\end{array}$ \\
\hline EPF-11 & $\begin{array}{c}0.00 \\
(0.00)\end{array}$ & $\begin{array}{c}0.00 \\
(0.00)\end{array}$ & $\begin{array}{c}0.00 \\
(0.00)\end{array}$ & $\begin{array}{c}16.00 \\
(23.58)\end{array}$ & $\begin{array}{c}16.00 \\
(23.56)\end{array}$ & $\begin{array}{c}48.00 \\
(43.85)\end{array}$ & $\begin{array}{c}48.00 \\
(43.85)\end{array}$ & $\begin{array}{c}68.00 \\
(55.55)\end{array}$ & $\begin{array}{c}68.00 \\
(55.55)\end{array}$ & $\begin{array}{c}86.00 \\
(68.03)\end{array}$ \\
\hline EPF-12 & $\begin{array}{c}0.00 \\
(0.00)\end{array}$ & $\begin{array}{c}0.00 \\
(0.00)\end{array}$ & $\begin{array}{c}0.00 \\
(0.00)\end{array}$ & $\begin{array}{c}14.00 \\
(21.97)\end{array}$ & $\begin{array}{c}22.00 \\
(27.97)\end{array}$ & $\begin{array}{c}40.00 \\
(39.23)\end{array}$ & $\begin{array}{c}40.00 \\
(39.23)\end{array}$ & $\begin{array}{c}68.00 \\
(55.55)\end{array}$ & $\begin{array}{c}78.00 \\
(62.03)\end{array}$ & $\begin{array}{c}78.00 \\
(62.03)\end{array}$ \\
\hline EPF-13 & $\begin{array}{c}0.00 \\
(0.00)\end{array}$ & $\begin{array}{c}0.00 \\
(0.00)\end{array}$ & $\begin{array}{c}0.00 \\
(0.00)\end{array}$ & $\begin{array}{c}0.00 \\
(0.00)\end{array}$ & $\begin{array}{c}14.00 \\
(21.97)\end{array}$ & $\begin{array}{c}22.00 \\
(27.97)\end{array}$ & $\begin{array}{c}28.00 \\
(31.95)\end{array}$ & $\begin{array}{c}28.00 \\
(31.95)\end{array}$ & $\begin{array}{c}42.00 \\
(40.40)\end{array}$ & $\begin{array}{c}66.00 \\
(54.33)\end{array}$ \\
\hline EPF-14 & $\begin{array}{c}0.00 \\
(0.00)\end{array}$ & $\begin{array}{c}0.00 \\
(0.00)\end{array}$ & $\begin{array}{c}20.00 \\
(26.56)\end{array}$ & $\begin{array}{c}20.00 \\
(26.56)\end{array}$ & $\begin{array}{c}50.00 \\
(45.00)\end{array}$ & $\begin{array}{c}50.00 \\
(45.00)\end{array}$ & $\begin{array}{c}72.00 \\
(58.05)\end{array}$ & $\begin{array}{c}80.00 \\
(63.44)\end{array}$ & $\begin{array}{c}82.00 \\
(64.90)\end{array}$ & $\begin{array}{l}100.00 \\
(90.00)\end{array}$ \\
\hline EPF-15 & $\begin{array}{c}0.00 \\
(0.00)\end{array}$ & $\begin{array}{c}0.00 \\
(0.00)\end{array}$ & $\begin{array}{c}0.00 \\
(0.00)\end{array}$ & $\begin{array}{c}0.00 \\
(0.00)\end{array}$ & $\begin{array}{c}24.00 \\
(29.33)\end{array}$ & $\begin{array}{c}24.00 \\
(29.33)\end{array}$ & $\begin{array}{c}58.00 \\
(49.60)\end{array}$ & $\begin{array}{c}72.00 \\
(58.05)\end{array}$ & $\begin{array}{c}76.00 \\
(60.67)\end{array}$ & $\begin{array}{c}84.00 \\
(66.42)\end{array}$ \\
\hline SE & 0.00 & 0.00 & 0.81 & 0.81 & 0.93 & 1.24 & 1.45 & 1.64 & 1.66 & 2.12 \\
\hline CD@5\% & 0.00 & 0.00 & 2.44 & 2.45 & 2.83 & 3.76 & 4.37 & 4.96 & 5.02 & 6.40 \\
\hline
\end{tabular}

Figures in parenthesis are arc sin values DAT= Day After Treatment 
Int.J.Curr.Microbiol.App.Sci (2019) 8(3): 2166-2175

Table.3 The virulence of entomopathogenic fungi isolates at $10^{6}$ spore concentration

\begin{tabular}{|c|c|c|c|c|c|c|c|c|c|c|}
\hline \multirow[t]{2}{*}{ Isolate } & \multicolumn{10}{|c|}{ Per cent mortality at $10^{6}$ spore concentration } \\
\hline & 1DAT & 2DAT & 3DAT & 4DAT & 5DAT & 6DAT & 7DAT & 8DAT & 9DAT & 10DAT \\
\hline EPF-1 & $\begin{array}{c}0.00 \\
(0.00)\end{array}$ & $\begin{array}{c}0.00 \\
(0.00)\end{array}$ & $\begin{array}{c}0.00 \\
(0.00)\end{array}$ & $\begin{array}{c}0.00 \\
(0.00)\end{array}$ & $\begin{array}{c}14.00 \\
(21.97)\end{array}$ & $\begin{array}{c}14.00 \\
(21.97)\end{array}$ & $\begin{array}{c}14.00 \\
(21.97)\end{array}$ & $\begin{array}{c}38.00 \\
(38.05)\end{array}$ & $\begin{array}{c}38.00 \\
(38.05)\end{array}$ & $\begin{array}{c}44.00 \\
(41.55)\end{array}$ \\
\hline EPF-2 & $\begin{array}{c}0.00 \\
(0.00)\end{array}$ & $\begin{array}{c}0.00 \\
(0.00)\end{array}$ & $\begin{array}{c}0.00 \\
(0.00)\end{array}$ & $\begin{array}{c}6.00 \\
(14.18)\end{array}$ & $\begin{array}{c}10.00 \\
(18.44)\end{array}$ & $\begin{array}{c}20.00 \\
(26.56)\end{array}$ & $\begin{array}{c}34.00 \\
(35.67)\end{array}$ & $\begin{array}{c}38.00 \\
(38.05)\end{array}$ & $\begin{array}{c}50.00 \\
(45.00)\end{array}$ & $\begin{array}{c}50.00 \\
(45.00)\end{array}$ \\
\hline EPF-3 & $\begin{array}{c}0.00 \\
(0.00)\end{array}$ & $\begin{array}{c}0.00 \\
(0.00)\end{array}$ & $\begin{array}{c}0.00 \\
(0.00)\end{array}$ & $\begin{array}{c}16.00 \\
(23.58)\end{array}$ & $\begin{array}{c}16.00 \\
(23.58)\end{array}$ & $\begin{array}{c}40.00 \\
(39.23)\end{array}$ & $\begin{array}{c}40.00 \\
(39.23)\end{array}$ & $\begin{array}{c}46.00 \\
(42.71)\end{array}$ & $\begin{array}{c}46.00 \\
(42.71)\end{array}$ & $\begin{array}{c}50.00 \\
(45.00)\end{array}$ \\
\hline EPF-4 & $\begin{array}{c}0.00 \\
(0.00)\end{array}$ & $\begin{array}{c}0.00 \\
(0.00)\end{array}$ & $\begin{array}{c}0.00 \\
(0.00)\end{array}$ & $\begin{array}{c}0.00 \\
(0.00)\end{array}$ & $\begin{array}{c}12.00 \\
(20.27)\end{array}$ & $\begin{array}{c}12.00 \\
(20.27)\end{array}$ & $\begin{array}{c}20.00 \\
(26.56)\end{array}$ & $\begin{array}{c}34.00 \\
(35.67)\end{array}$ & $\begin{array}{c}40.00 \\
(39.23)\end{array}$ & $\begin{array}{c}40.00 \\
(39.23)\end{array}$ \\
\hline EPF-5 & $\begin{array}{c}0.00 \\
(0.00)\end{array}$ & $\begin{array}{c}0.00 \\
(0.00)\end{array}$ & $\begin{array}{c}0.00 \\
(0.00)\end{array}$ & $\begin{array}{c}0.00 \\
(0.00)\end{array}$ & $\begin{array}{c}14.00 \\
(21.97)\end{array}$ & $\begin{array}{c}14.00 \\
(21.97)\end{array}$ & $\begin{array}{c}32.00 \\
(34.45)\end{array}$ & $\begin{array}{c}32.00 \\
(34.45)\end{array}$ & $\begin{array}{c}32.00 \\
(34.45)\end{array}$ & $\begin{array}{c}46.00 \\
(42.71)\end{array}$ \\
\hline EPF-6 & $\begin{array}{c}0.00 \\
(0.00)\end{array}$ & $\begin{array}{c}0.00 \\
(0.00)\end{array}$ & $\begin{array}{c}0.00 \\
(0.00)\end{array}$ & $\begin{array}{c}6.00 \\
(14.18)\end{array}$ & $\begin{array}{c}10.00 \\
(18.44)\end{array}$ & $\begin{array}{c}18.00 \\
(25.10)\end{array}$ & $\begin{array}{c}24.00 \\
(29.33)\end{array}$ & $\begin{array}{c}30.00 \\
(33.21)\end{array}$ & $\begin{array}{c}46.00 \\
(42.71)\end{array}$ & $\begin{array}{c}46.00 \\
(42.71)\end{array}$ \\
\hline EPF-7 & $\begin{array}{c}0.00 \\
(0.00)\end{array}$ & $\begin{array}{c}0.00 \\
(0.00)\end{array}$ & $\begin{array}{c}0.00 \\
(0.00)\end{array}$ & $\begin{array}{c}8.00 \\
(16.43)\end{array}$ & $\begin{array}{c}10.00 \\
(18.44)\end{array}$ & $\begin{array}{c}16.00 \\
(23.58)\end{array}$ & $\begin{array}{c}26.00 \\
(30.66)\end{array}$ & $\begin{array}{c}50.00 \\
(45.00)\end{array}$ & $\begin{array}{c}54.00 \\
(47.29)\end{array}$ & $\begin{array}{c}54.00 \\
(47.29)\end{array}$ \\
\hline EPF-8 & $\begin{array}{c}0.00 \\
(0.00)\end{array}$ & $\begin{array}{c}0.00 \\
(0.00)\end{array}$ & $\begin{array}{c}0.00 \\
(0.00)\end{array}$ & $\begin{array}{c}0.00 \\
(0.00)\end{array}$ & $\begin{array}{c}10.00 \\
(18.44)\end{array}$ & $\begin{array}{c}20.00 \\
(26.56)\end{array}$ & $\begin{array}{c}26.00 \\
(30.66)\end{array}$ & $\begin{array}{c}32.00 \\
(34.45)\end{array}$ & $\begin{array}{c}50.00 \\
(45.00)\end{array}$ & $\begin{array}{c}66.00 \\
(54.33)\end{array}$ \\
\hline EPF-9 & $\begin{array}{c}0.00 \\
(0.00)\end{array}$ & $\begin{array}{c}0.00 \\
(0.00)\end{array}$ & $\begin{array}{c}0.00 \\
(0.00)\end{array}$ & $\begin{array}{c}0.00 \\
(0.00)\end{array}$ & $\begin{array}{c}14.00 \\
(21.97)\end{array}$ & $\begin{array}{c}24.00 \\
(29.33)\end{array}$ & $\begin{array}{c}26.00 \\
(30.66)\end{array}$ & $\begin{array}{c}38.00 \\
(38.05)\end{array}$ & $\begin{array}{c}44.00 \\
(41.55)\end{array}$ & $\begin{array}{c}46.00 \\
(42.71)\end{array}$ \\
\hline EPF-10 & $\begin{array}{c}0.00 \\
(0.00)\end{array}$ & $\begin{array}{c}0.00 \\
(0.00)\end{array}$ & $\begin{array}{c}0.00 \\
(0.00)\end{array}$ & $\begin{array}{c}0.00 \\
(0.00)\end{array}$ & $\begin{array}{c}10.00 \\
(18.44)\end{array}$ & $\begin{array}{c}16.00 \\
(23.58)\end{array}$ & $\begin{array}{c}22.00 \\
(27.97)\end{array}$ & $\begin{array}{c}32.00 \\
(34.45)\end{array}$ & $\begin{array}{c}48.00 \\
(43.85)\end{array}$ & $\begin{array}{c}66.00 \\
(54.33)\end{array}$ \\
\hline EPF-11 & $\begin{array}{c}0.00 \\
(0.00)\end{array}$ & $\begin{array}{c}0.00 \\
(0.00)\end{array}$ & $\begin{array}{c}0.00 \\
(0.00)\end{array}$ & $\begin{array}{c}0.00 \\
(0.00)\end{array}$ & $\begin{array}{c}14.00 \\
(21.97)\end{array}$ & $\begin{array}{c}14.00 \\
(21.97)\end{array}$ & $\begin{array}{c}46.00 \\
(42.71)\end{array}$ & $\begin{array}{c}46.00 \\
(42.71)\end{array}$ & $\begin{array}{c}56.00 \\
(48.45)\end{array}$ & $\begin{array}{c}66.00 \\
(54.33)\end{array}$ \\
\hline EPF-12 & $\begin{array}{c}0.00 \\
(0.00)\end{array}$ & $\begin{array}{c}0.00 \\
(0.00)\end{array}$ & $\begin{array}{c}0.00 \\
(0.00)\end{array}$ & $\begin{array}{c}14.00 \\
(21.97)\end{array}$ & $\begin{array}{c}20.00 \\
(26.56)\end{array}$ & $\begin{array}{c}40.00 \\
(39.23)\end{array}$ & $\begin{array}{c}40.00 \\
(39.23)\end{array}$ & $\begin{array}{c}56.00 \\
(48.45)\end{array}$ & $\begin{array}{c}68.00 \\
(55.55)\end{array}$ & $\begin{array}{c}68.00 \\
(55.55)\end{array}$ \\
\hline EPF-13 & $\begin{array}{c}0.00 \\
(0.00)\end{array}$ & $\begin{array}{c}0.00 \\
(0.00)\end{array}$ & $\begin{array}{c}0.00 \\
(0.00)\end{array}$ & $\begin{array}{c}0.00 \\
(0.00)\end{array}$ & $\begin{array}{c}0.00 \\
(0.00)\end{array}$ & $\begin{array}{c}12.00 \\
(20.27)\end{array}$ & $\begin{array}{c}12.00 \\
(20.27)\end{array}$ & $\begin{array}{c}22.00 \\
(27.97)\end{array}$ & $\begin{array}{c}36.00 \\
(36.87)\end{array}$ & $\begin{array}{c}54.00 \\
(47.29)\end{array}$ \\
\hline EPF-14 & $\begin{array}{c}0.00 \\
(0.00)\end{array}$ & $\begin{array}{c}0.00 \\
(0.00)\end{array}$ & $\begin{array}{c}0.00 \\
(0.00)\end{array}$ & $\begin{array}{c}0.00 \\
(0.00)\end{array}$ & $\begin{array}{c}10.00 \\
(18.44)\end{array}$ & $\begin{array}{c}40.00 \\
(39.23)\end{array}$ & $\begin{array}{c}54.00 \\
(47.29)\end{array}$ & $\begin{array}{c}72.00 \\
(58.05)\end{array}$ & $\begin{array}{c}82.00 \\
(64.90)\end{array}$ & $\begin{array}{c}88.00 \\
(69.73)\end{array}$ \\
\hline EPF-15 & $\begin{array}{c}0.00 \\
(0.00)\end{array}$ & $\begin{array}{c}0.00 \\
(0.00)\end{array}$ & $\begin{array}{c}0.00 \\
(0.00)\end{array}$ & $\begin{array}{c}0.00 \\
(0.00)\end{array}$ & $\begin{array}{c}10.00 \\
(18.44)\end{array}$ & $\begin{array}{c}24.00 \\
(29.33)\end{array}$ & $\begin{array}{c}50.00 \\
(45.00)\end{array}$ & $\begin{array}{c}60.00 \\
(50.77)\end{array}$ & $\begin{array}{c}72.00 \\
(58.05)\end{array}$ & $\begin{array}{c}80.00 \\
(63.44)\end{array}$ \\
\hline SE & 0.00 & 0.00 & 0.00 & 3.92 & 1.45 & 0.91 & 1.13 & 1.25 & 1.21 & 1.64 \\
\hline CD@5\% & 0.00 & 0.00 & 0.00 & 11.82 & 4.38 & 2.75 & 3.42 & 3.79 & 3.65 & 4.96 \\
\hline
\end{tabular}

Figures in parenthesis are arc sin values DAT= Day After Treatment 
Table.4 The virulence of entomopathogenic fungi isolates at $10^{9}$ spore concentration

\begin{tabular}{|c|c|c|c|c|c|c|c|c|c|c|}
\hline \multirow[t]{2}{*}{ Isolates } & \multicolumn{10}{|c|}{ Per cent mortality at $\mathbf{1 0}^{9}$ spore concentration } \\
\hline & 1DAT & 2DAT & 3DAT & 4DAT & 5DAT & 6DAT & 7DAT & 8DAT & 9DAT & 10DAT \\
\hline EPF-1 & $\begin{array}{c}0.00 \\
(0.00)\end{array}$ & $\begin{array}{c}0.00 \\
(0.00)\end{array}$ & $\begin{array}{c}0.00 \\
(0.00)\end{array}$ & $\begin{array}{c}0.00 \\
(0.00)\end{array}$ & $\begin{array}{c}0.00 \\
(0.00)\end{array}$ & $\begin{array}{c}0.00 \\
(0.00)\end{array}$ & $\begin{array}{c}14.00 \\
(21.97)\end{array}$ & $\begin{array}{c}28.00 \\
(31.95)\end{array}$ & $\begin{array}{c}28.00 \\
(31.95)\end{array}$ & $\begin{array}{c}42.00 \\
(40.40)\end{array}$ \\
\hline EPF-2 & $\begin{array}{c}0.00 \\
(0.00)\end{array}$ & $\begin{array}{c}0.00 \\
(0.00)\end{array}$ & $\begin{array}{c}0.00 \\
(0.00)\end{array}$ & $\begin{array}{c}0.00 \\
(0.00)\end{array}$ & $\begin{array}{c}6.00 \\
(14.18)\end{array}$ & $\begin{array}{c}18.00 \\
(25.10)\end{array}$ & $\begin{array}{c}26.00 \\
(30.66)\end{array}$ & $\begin{array}{c}28.00 \\
(31.95)\end{array}$ & $\begin{array}{c}28.00 \\
(31.95)\end{array}$ & $\begin{array}{c}36.00 \\
(36.87)\end{array}$ \\
\hline EPF-3 & $\begin{array}{c}0.00 \\
(0.00)\end{array}$ & $\begin{array}{c}0.00 \\
(0.00)\end{array}$ & $\begin{array}{c}0.00 \\
(0.00)\end{array}$ & $\begin{array}{c}0.00 \\
(0.00)\end{array}$ & $\begin{array}{c}14.00 \\
(21.97)\end{array}$ & $\begin{array}{c}14.00 \\
(21.97)\end{array}$ & $\begin{array}{c}32.00 \\
(34.45)\end{array}$ & $\begin{array}{c}32.00 \\
(34.45)\end{array}$ & $\begin{array}{c}44.00 \\
(41.55)\end{array}$ & $\begin{array}{c}44.00 \\
(41.55)\end{array}$ \\
\hline EPF-4 & $\begin{array}{c}0.00 \\
(0.00)\end{array}$ & $\begin{array}{c}0.00 \\
(0.00)\end{array}$ & $\begin{array}{c}0.00 \\
(0.00)\end{array}$ & $\begin{array}{c}0.00 \\
(0.00)\end{array}$ & $\begin{array}{c}0.00 \\
(0.00)\end{array}$ & $\begin{array}{c}12.00 \\
(20.27)\end{array}$ & $\begin{array}{c}12.00 \\
(20.27)\end{array}$ & $\begin{array}{c}28.00 \\
(31.95)\end{array}$ & $\begin{array}{c}28.00 \\
(31.95)\end{array}$ & $\begin{array}{c}36.00 \\
(36.87)\end{array}$ \\
\hline EPF-5 & $\begin{array}{c}0.00 \\
(0.00)\end{array}$ & $\begin{array}{c}0.00 \\
(0.00)\end{array}$ & $\begin{array}{c}0.00 \\
(0.00)\end{array}$ & $\begin{array}{c}4.00 \\
(11.54)\end{array}$ & $\begin{array}{c}4.00 \\
(11.54)\end{array}$ & $\begin{array}{c}10.00 \\
(18.44)\end{array}$ & $\begin{array}{c}10.00 \\
(18.44)\end{array}$ & $\begin{array}{c}20.00 \\
(26.56)\end{array}$ & $\begin{array}{c}20.00 \\
(26.56)\end{array}$ & $\begin{array}{c}26.00 \\
(30.66)\end{array}$ \\
\hline EPF-6 & $\begin{array}{c}0.00 \\
(0.00)\end{array}$ & $\begin{array}{c}0.00 \\
(0.00)\end{array}$ & $\begin{array}{c}0.00 \\
(0.00)\end{array}$ & $\begin{array}{c}0.00 \\
(0.00)\end{array}$ & $\begin{array}{c}6.00 \\
(14.18)\end{array}$ & $\begin{array}{c}10.00 \\
(18.44)\end{array}$ & $\begin{array}{c}10.00 \\
(18.44)\end{array}$ & $\begin{array}{c}24.00 \\
(29.33)\end{array}$ & $\begin{array}{c}24.00 \\
(29.33)\end{array}$ & $\begin{array}{c}34.00 \\
(35.67)\end{array}$ \\
\hline EPF-7 & $\begin{array}{c}0.00 \\
(0.00)\end{array}$ & $\begin{array}{c}0.00 \\
(0.00)\end{array}$ & $\begin{array}{c}0.00 \\
(0.00)\end{array}$ & $\begin{array}{c}0.00 \\
(0.00)\end{array}$ & $\begin{array}{c}8.00 \\
(16.43)\end{array}$ & $\begin{array}{c}10.00 \\
(18.44)\end{array}$ & $\begin{array}{c}26.00 \\
(30.66)\end{array}$ & $\begin{array}{c}26.00 \\
(30.66)\end{array}$ & $\begin{array}{c}26.00 \\
(30.66)\end{array}$ & $\begin{array}{c}30.00 \\
(33.21)\end{array}$ \\
\hline EPF-8 & $\begin{array}{c}0.00 \\
(0.00)\end{array}$ & $\begin{array}{c}0.00 \\
(0.00)\end{array}$ & $\begin{array}{c}0.00 \\
(0.00)\end{array}$ & $\begin{array}{c}0.00 \\
(0.00)\end{array}$ & $\begin{array}{c}0.00 \\
(0.00)\end{array}$ & $\begin{array}{c}16.00 \\
(23.58)\end{array}$ & $\begin{array}{c}22.00 \\
(27.97)\end{array}$ & $\begin{array}{c}32.00 \\
(34.45)\end{array}$ & $\begin{array}{c}38.00 \\
(38.06)\end{array}$ & $\begin{array}{c}46.00 \\
(42.71)\end{array}$ \\
\hline EPF-9 & $\begin{array}{c}0.00 \\
(0.00)\end{array}$ & $\begin{array}{c}0.00 \\
(0.00)\end{array}$ & $\begin{array}{c}0.00 \\
(0.00)\end{array}$ & $\begin{array}{c}0.00 \\
(0.00)\end{array}$ & $\begin{array}{c}10.00 \\
(18.44)\end{array}$ & $\begin{array}{c}10.00 \\
(18.44)\end{array}$ & $\begin{array}{c}20.00 \\
(26.56)\end{array}$ & $\begin{array}{c}20.00 \\
(26.56)\end{array}$ & $\begin{array}{c}38.00 \\
(38.06)\end{array}$ & $\begin{array}{c}44.00 \\
(41.55)\end{array}$ \\
\hline EPF-10 & $\begin{array}{c}0.00 \\
(0.00)\end{array}$ & $\begin{array}{c}0.00 \\
(0.00)\end{array}$ & $\begin{array}{c}0.00 \\
(0.00)\end{array}$ & $\begin{array}{c}0.00 \\
(0.00)\end{array}$ & $\begin{array}{c}0.00 \\
(0.00)\end{array}$ & $\begin{array}{c}8.00 \\
(16.43)\end{array}$ & $\begin{array}{c}14.00 \\
(21.97)\end{array}$ & $\begin{array}{c}14.00 \\
(21.97)\end{array}$ & $\begin{array}{c}28.00 \\
(31.95)\end{array}$ & $\begin{array}{c}48.00 \\
(43.85)\end{array}$ \\
\hline EPF-11 & $\begin{array}{c}0.00 \\
(0.00)\end{array}$ & $\begin{array}{c}0.00 \\
(0.00)\end{array}$ & $\begin{array}{c}0.00 \\
(0.00)\end{array}$ & $\begin{array}{c}0.00 \\
(0.00)\end{array}$ & $\begin{array}{c}0.00 \\
(0.00)\end{array}$ & $\begin{array}{c}8.00 \\
(16.43)\end{array}$ & $\begin{array}{c}8.00 \\
(16.43)\end{array}$ & $\begin{array}{c}8.00 \\
(16.43)\end{array}$ & $\begin{array}{c}16.00 \\
(23.58)\end{array}$ & $\begin{array}{c}32.00 \\
(34.45)\end{array}$ \\
\hline EPF-12 & $\begin{array}{c}0.00 \\
(0.00)\end{array}$ & $\begin{array}{c}0.00 \\
(0.00)\end{array}$ & $\begin{array}{c}0.00 \\
(0.00)\end{array}$ & $\begin{array}{c}14.00 \\
(21.97)\end{array}$ & $\begin{array}{c}14.00 \\
(21.97)\end{array}$ & $\begin{array}{c}22.00 \\
(27.97)\end{array}$ & $\begin{array}{c}34.00 \\
(35.67)\end{array}$ & $\begin{array}{c}34.00 \\
(35.67)\end{array}$ & $\begin{array}{c}42.00 \\
(40.40)\end{array}$ & $\begin{array}{c}46.00 \\
(42.71)\end{array}$ \\
\hline EPF-13 & $\begin{array}{c}0.00 \\
(0.00)\end{array}$ & $\begin{array}{c}0.00 \\
(0.00)\end{array}$ & $\begin{array}{c}0.00 \\
(0.00)\end{array}$ & $\begin{array}{c}0.00 \\
(0.00)\end{array}$ & $\begin{array}{c}0.00 \\
(0.00)\end{array}$ & $\begin{array}{c}0.00 \\
(0.00)\end{array}$ & $\begin{array}{c}8.00 \\
(16.43)\end{array}$ & $\begin{array}{c}8.00 \\
(16.43)\end{array}$ & $\begin{array}{c}28.00 \\
(31.95)\end{array}$ & $\begin{array}{c}34.00 \\
(35.67)\end{array}$ \\
\hline EPF-14 & $\begin{array}{c}0.00 \\
(0.00)\end{array}$ & $\begin{array}{c}0.00 \\
(0.00)\end{array}$ & $\begin{array}{c}0.00 \\
(0.00)\end{array}$ & $\begin{array}{c}8.00 \\
(16.43)\end{array}$ & $\begin{array}{c}10.00 \\
(18.44)\end{array}$ & $\begin{array}{c}36.00 \\
(36.87)\end{array}$ & $\begin{array}{c}36.00 \\
(36.87)\end{array}$ & $\begin{array}{c}36.00 \\
(36.87)\end{array}$ & $\begin{array}{c}68.00 \\
(55.55)\end{array}$ & $\begin{array}{c}74.00 \\
(59.34)\end{array}$ \\
\hline EPF-15 & $\begin{array}{c}0.00 \\
(0.00)\end{array}$ & $\begin{array}{c}0.00 \\
(0.00)\end{array}$ & $\begin{array}{c}0.00 \\
(0.00)\end{array}$ & $\begin{array}{c}0.00 \\
(0.00)\end{array}$ & $\begin{array}{c}6.00 \\
(14.18)\end{array}$ & $\begin{array}{c}6.00 \\
(14.18)\end{array}$ & $\begin{array}{c}30.00 \\
(33.21)\end{array}$ & $\begin{array}{c}30.00 \\
(33.21)\end{array}$ & $\begin{array}{c}60.00 \\
(50.77)\end{array}$ & $\begin{array}{c}60.00 \\
(50.77)\end{array}$ \\
\hline SE & 0.00 & 0.00 & 0.00 & 0.42 & 0.88 & 1.22 & 1.18 & 0.76 & 0.62 & 1.23 \\
\hline $\begin{array}{l}\text { CD } \\
@ 5 \%\end{array}$ & 0.00 & 0.00 & 0.00 & 1.28 & 2.67 & 3.76 & 3.58 & 2.12 & 1.88 & 3.72 \\
\hline
\end{tabular}

Figures in parenthesis are arc sin values DAT= Day After Treatment 
Fig. No. 1 The virulence of entomopathogenic fungi isolates

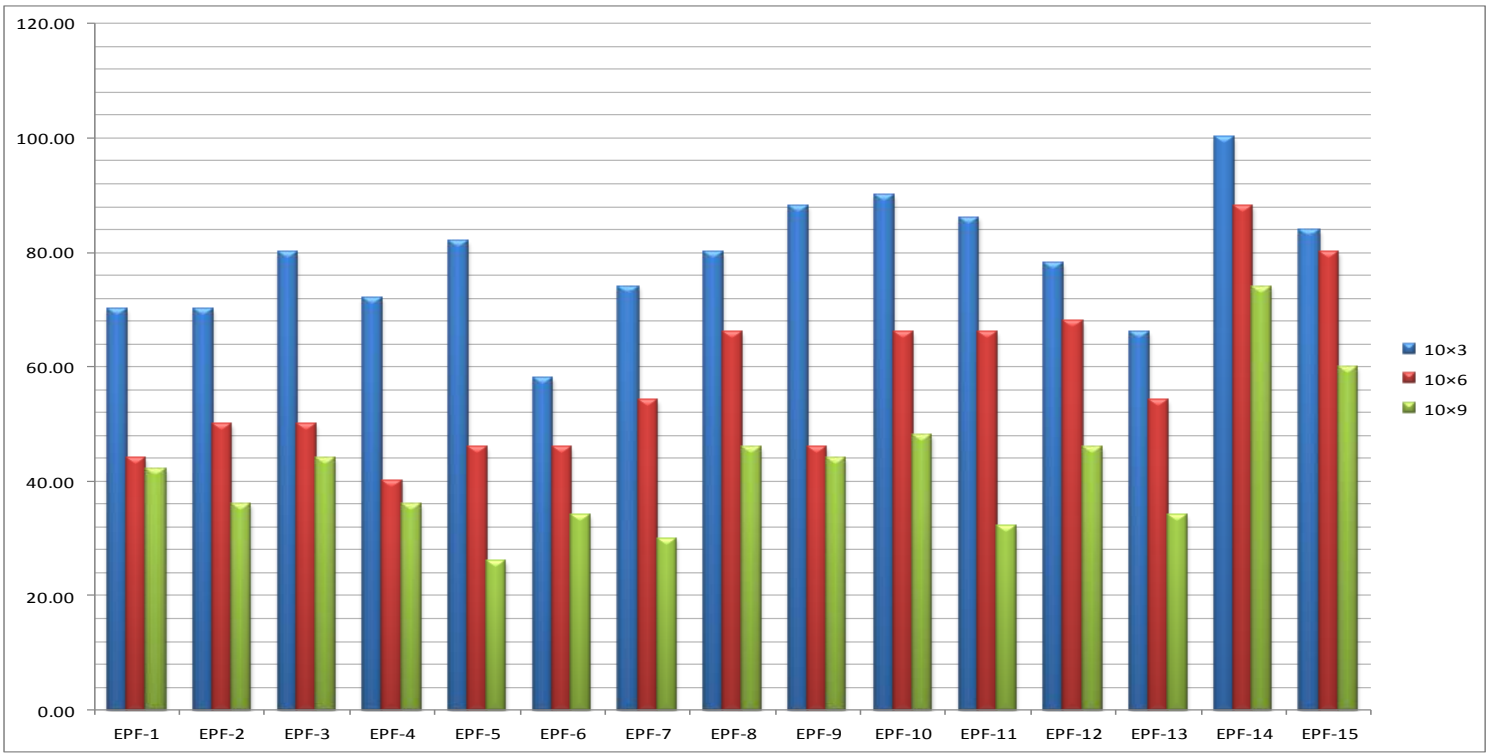

Among each isolate at $10^{-3}$ spores $/ \mathrm{ml}$ concentration the insect mortality was noted earlier compare to $10^{-6}$ and $10^{-9}$ spores $/ \mathrm{ml}$ concentration, while at $10^{-6}$ spores $/ \mathrm{ml}$ concentration the insect mortality was noted earlier compare to $10^{-9}$ spores $/ \mathrm{ml}$ concentration. At all the three-spore concentration the isolate EPF-14 had recorded the highest mealy bug's mortality compare to rest of isolates. Form the present research it was pointed out that at $10^{-3}$ spores $/ \mathrm{ml}$ concentration, the isolate EPF-14 recorded the highest mealy bugs mortality followed by EPF-3 and EPF-7 at 3 DAI means EPF-14 was the most aggressive isolate compare to the rest of the isolates (Table 2).

At 10 DAI, the isolate EPF-14 had recorded the highest mealy bugs mortality i.e. $100 \%$, $88 \%$ and $74 \%$ at $10^{-3}, 10^{-6}$ and $10^{-9}$ spores $/ \mathrm{ml}$ concentration respectively means the isolate EPF-14 was the most virulent isolate compare to the rest of the isolates. While, at $10^{-3}$ spores/ml concentration EPF-10 was next most virulent isolate compare to the rest of the isolates and at $10^{-6}$ and $10^{-9}$ spores $/ \mathrm{ml}$ concentration EPF-15 was next most virulent isolate compare to the rest of the isolates (Table 3).

Among the isolates of Aspergillus tamarii, the isolate EPF-7 was the most virulent against mealy bugs at $10^{-3}$ spores $/ \mathrm{ml}$ concentration and at $10^{-6}$ and $10^{-9}$ spores $/ \mathrm{ml}$ concentration the isolate EPF-1 was the most virulent. Among the isolates of Aspergillus niger, the isolate $\mathrm{EPF}-14$ was the most virulent isolate against mealy bugs at $10^{-3}$ spores $/ \mathrm{ml}$ concentration and at $10^{-6}$ and $10^{-9} \mathrm{spores} / \mathrm{ml}$ concentration the isolate EPF-2 was the most virulent isolate while, among the isolates of Aspergillus flavus, the isolate EPF-6 was the most virulent isolate at $10^{-3}, 10^{-6}$ and $10^{-9}$ spores/ml concentration.

Fungal isolates were isolated from the insect cadavers on PDA media and most of the isolates were resemble with Aspergillus spp. The selective media DOC2-50\% was used for the isolation of entomopathogenic fungi (Shin et al., 2010). Out of nineteen samples inoculated only fifteen samples showed the growth of fungus on DOC2-50\% selective media. 
The virulence studies of entomopathogenic fungi isolates revealed that, as the spore concentration increases from $10^{-3}$ to $10^{-9}$ the insect mortality decreases. Among all the fifteen isolates the highest insect mortality was observed at $10^{-3}$ spores $/ \mathrm{ml}$ concentration. At all the three-spore concentration the isolate EPF-14 had recorded the highest mealy bug's mortality followed by EPF-3 and EPF-7 at 3 DAI means EPF-14 was the most aggressive isolate compare to the rest of the isolates. Among the isolates of Aspergillus tamarii, the isolate EPF-7 was the most virulent against mealy bugs at $10^{-3}$ spores $/ \mathrm{ml}$ concentration and at $10^{-6}$ and $10^{-9}$ spores $/ \mathrm{ml}$ concentration the isolate EPF-1 was the most virulent. Similar task is carried out by Fawrou et al., (2014) in laboratory bioassay with five different concentrations of Aspergillus clavatus (Desmazières), Aspergillus flavus (Link) and Metarhizium anisopliae ((Metschnikoff) Sorokin) spores against the pea aphid, Acyrthosiphon pisum (Harris). Aspergillus isolates induced higher mortalities than $M$. anisopliae. Lethal concentrations (LC50 and LC90) were $1.23 \times 103$ and $1.34 \times$ 107 spores/ml for A. flavus, $4.95 \times 102$ and $5.65 \times 107$ spores $/ \mathrm{ml}$ for $A$. clavatus, and 3.67 $\times 103$ and $9.71 \times 107$ spores $/ \mathrm{ml}$ for $M$. anisopliae after 5 days of treatment. Mycelia development and sporulation on adult cadavers were observed at $48 \mathrm{hr}$ after incubation.

\section{References}

Abdalla, A. S. and Naiema, E. G. 2013. Isolation and characterization of new entomopathogenic fungi from the Sudan. International Journal of Science Innovations and Discoveries. 3 (3):326-329.

Ali-Shtayeh, M.S., Mara, A.B.B. and Jamous, R. M. 2002. Distribution, occurrence and characterization of entomopathogenic fungi in agricultural soil in the Palestinian area. Mycopathologia. 156:235-244.

Alexopoulos, C. J., Mims, C. W. and Blackwell, M. 1996. Introductory Mycology, 4th edition, New York, John Willey \& Sons. pp. 105.

Anonymous .2015. http: //www. mycology. adelaide.edu.au / fungal description.

Berger, E.W. 1921. Natural enemies of scale insects and whiteflies in Florida. Florida State. Plant Breeding Quartely Bulletin. 5: 141-154.

Burges, H.D. 1981. Progress in the microbial control of pests in Microbial Control of Pests and Plant Diseases. (Ed. H.D. Burges), Academic Press, London, pp.1-6.

Deshpande, M. V., Chandele, A. G., Nahar, A., Hadapad, A., Patil, A. G., Ghorpade, V., Keller, S. and Tour, U. 2001. Entomopathogenic fungi mycoinsecticides useful against lepidopteron pests in pulses. 8th European meeting of IOBC-WPRS worker group "Insect pathogen and insect parasitic nematode", held at Atlons, Greecas, pp. 17.

Fawrou, S., Thomas, B., Slimane, B., Jean, Y. Z.A. and Mady, N. 2014. Effect of entomopathogenic fungi Aspergillus strain against the pea aphid, Acyrthosophon pisum (Hemiptera-A. phididae). Applied entomology and zoology. 49(3) : 10-14.

Gupta, M., Kumari, M. and Ruby, G. 2012. Effect of various media types on the rate of growth of Aspergillus niger. Indian Journal of Fundamental and Applied Life Sciences ISSN. 2 (2):2231-6345.

Hajek, A.E. And Ledger, R.J. 1994. Interaction between fungal pathogens and insect hosts. Annual Review of Entomology. 39: 293-322.

Hina, A., Saleem, S. and Syeda, Q. U. N. 2013. Morphological identification of 
Aspergillus species from the soil of Larkana district (Sindh-Pakistan). Asian Journal of Agriculture Biology. 1(3):105-117.

Kirsten, F., Ge, R. F., Annette, B. J. and William, O.H. H. 2014. The distribution of Aspergillus spp. opportunistic parasites in hives and their pathogenicity to honey bees. Veterinary Microbiology. 169: 203210

Klich, M.A. and Pitt. J.1. 1988 . Differentiation of Aspergillus flavus from $A$. parasiticus and other closely related species. Trans. Brit. Mycol. Soc. 91: 99-108.

Lazgeen, H. A., Raed, A. H. and Samir, K.A. 2011. Association of Entomopathogenic and Other Opportunistic Fungi with Insects in Dormant Locations. Jordan Journal of Biological Sciences. 4: 1-170.

Maddox, J. V. 1994. Insect pathogens as biological control agents. In Introduction to Insect pests Management, (eds. Metcalf R.L. and Lukmann, W.H.). John Wiley and Sons Inc. Publ. New York, pp. 199. 244.

Mahapatro, G. K. 2007. Pesticides and technology 'Emerging trend in India' (Led paper) Souvenir in National symposium on sustainable pest management. Society of Plant Protection and Environment OUANT, Bhubaneshwar, India. pp.120.

Maryam, N. A., Hassan. A., Sohrab, I. and Rasoul, Z. 2014. Isolation and characterization of entomopathogenic fungi from hibernating sites of Sunn Pest (Eurygaster integriceps) on Ilam Mountains, Iran. International Journal of Current Microbiology and Applied Sciences. 3(12): 314-325.

McClenny, N. 2005. Laboratory detection and identification of Aspergillus species by microscopic observation and culture: the traditional approach. Journal of Medical and Veterinary Mycology. 43: S125-S128.

Nyongesa, B. W., Sheila, O., Vincent, A. 2015. Identification Key for Aspergillus species Isolated from Maize and Soil of Nandi County, Kenya. Advances in Microbiology. 5:205-229.

Odhiambo, B.O., Hunja, M. and Isabel, N.W.2013. Isolation and characterization of Aflatoxigenic Aspergillus species from maize and soil samples from selected counties of Kenya. African Journal of Microbiology Research. 7(34):43794388.

Pasaru, F., Alam, A., Tutik, K., Mahfudz. and Shahabuddin. 2014. Prospective of entomopathogenic fungi associated with Helopeltis spp. (Hemipter: Miridae) on cacao plantation. International Journal of Current Research and Academic Review. 2(11):227-234.

Pasteur, L. 1874. Observations (au sujet des conclusions de M. Dumas) relatives au phylloxera. Comptes rendus hebdomadaires des seances de 1 Academie des Sciences. 79 : 12331234.

Phadke, C. H. and Rao, V. G. 1978. Studies on the entomogenous fungus, $N$. rileyi. Current Science. 47 : 511-512.

Pitt, J.I., Hocking, A.D. and Glenn, D.R., 1983. An improved medium for detection of Aspergillus flavus and A. parasiticus. Journal of Applied Bacteriology. 54:109-114.

Raper, K.B. and Fennell, D. 1965 . The genus Aspergillus. Williams and Wilkins Company.

Sharma, C. S. 1999. Pesticides status in India, Special Issue Pestology. 2: 3-5.

Sharma, G. and Pandey, R. R. 2010. Influence 
of culture media on growth, colony character and sporulation of fungi isolated from decaying vegetable wastes. Journal of Yeast and Fungal Research. 1(8):157 - 164.

Shin, T.Y., Jae -Bang, C., Sung- Min, B., Hyun-Na, K. and Soo-Dong, D. 2010. Study on selective media for Isolation of entomopathogenic fungi. International Journal of Industrial Entomology. 20(1):7-12.

Steinhaus, E. 1956. Microbial control - the emergence of an idea. A brief history of insect pathology through the nineteenth century. Hilgardia. 26: 107160.

Stephenson, L. W. and Russell T.E. 1974. The association of Aspergillus flavus with hemipterous and other insects infesting cotton bracts and foliage.
Phytopathology. 64: 1S02-IS06.

Ulhan, S., Rasime, D., Ahmet, A. and Cengiz, B. E. K. 2006. Turkistan Journal of Botany. 30:95-104.

Wazeer, A. H., Lazgeen, H. A. and Samir, K. A. 2012. Occurrence of entomopathogenic and other opportunistic fungi in soil collected from insect hibernation sites and evaluation of their entomopathogenic potential. Bull. Iraq National Historical Museum. 12 (1): 19-27.

Zain, M.E., Razak, A. A., El-Sheikh, H.H., Soliman, H.G. and Khalil, A.M. 2009. Influence of growth medium on diagnostic characters of Aspergillus and Penicillium species. African Journal of Microbiology Research. 3 (5): 280-286.

\section{How to cite this article:}

Sable, S.B., P.B. Deore, V.R. Bangar and Kurhade, K.C. 2019 Effect of Different Spore Concentration of Entomopathogenic Fungi Isolates against Mealy Bug Maconellicocus hirsutus. Int.J.Curr.Microbiol.App.Sci. 8(03): 2166-2175.

doi: https://doi.org/10.20546/ijcmas.2019.803.259 